\title{
MT1G Gene
}

National Cancer Institute

\section{Source}

National Cancer Institute. MT1G Gene. NCI Thesaurus. Code C105397.

This gene is involved in chelation. 